\title{
Data-centric disambiguation for data transformation with programming-by-example
}

\author{
Minori Narita \\ University of Massachusetts Amherst \\ Massachusetts, USA \\ mnarita@umass.edu \\ Yi Lu \\ The University of Tokyo \\ Tokyo, Japan
}

\author{
Nolwen Maudet \\ University of Strasbourg \\ Strasbourg, France \\ Takeo Igarashi \\ The University of Tokyo \\ Tokyo, Japan
}

\begin{abstract}
Programming-by-example (PBE), can be a powerful tool to reduce manual work in repetitive data transformation tasks. However, few examples often leave ambiguity and may cause undesirable data transformation by the system. This ambiguity can be resolved by allowing the user to directly edit the synthesized programs; however, this is difficult for non-programmers. Here, we present a novel approach: data-centric disambiguation for data transformation, where users resolve the ambiguity in data transformation by examining and modifying the output rather than the program. The key idea is to focus on the given set of data the user wants to transform instead of pursuing the synthesized program's generality or completeness. Our system provides visualization and interaction methods that allow users to efficiently examine and fix the transformed outputs, which is much simpler than understanding and modifying the program itself. The user study suggests that our system can successfully help non-programmers to more easily and efficiently process data.
\end{abstract}

\section{CCS CONCEPTS}

•Human-centered computing $\rightarrow$ User interface programming.

\section{KEYWORDS}

Programming-by-example, User interface, Visualization

\section{ACM Reference Format:}

Minori Narita, Nolwen Maudet, Yi Lu, and Takeo Igarashi. 2021. Data-centric disambiguation for data transformation with programming-by-example. In 26th International Conference on Intelligent User Interfaces (IUI '21),April 14-17, 2021, College Station, TX, USA. ACM, New York, NY, USA, 10 pages. https://doi.org/10.1145/3397481.3450680

\footnotetext{
Permission to make digital or hard copies of all or part of this work for personal or classroom use is granted without fee provided that copies are not made or distributed for profit or commercial advantage and that copies bear this notice and the full citation on the first page. Copyrights for components of this work owned by others than ACM must be honored. Abstracting with credit is permitted. To copy otherwise, or republish, to post on servers or to redistribute to lists, requires prior specific permission and/or a fee. Request permissions from permissions@acm.org.

IUI '21, April 14-17, 2021, College Station, TX, USA

(C) 2021 Association for Computing Machinery.

ACM ISBN 978-1-4503-8017-1/21/04 ..\$15.00

https://doi.org/10.1145/3397481.3450680
}

\section{INTRODUCTION}

Data transformation is one of the most critical aspects of data analysis $[14,16,18,26]$. According to Wind [26], the data analysis process is divided into several steps: data preparation, feature generation and transformation, modeling, and operationalization. In particular, feature generation and transformation have significant impacts on the resultant prediction accuracy. For example, in Kaggle ${ }^{1}$, a data science competition website, the models and algorithms used by the winners are often rather simple; in such cases, feature engineering is the decisive factor. Feature engineering is the process of transforming original features into a new feature that more accurately or succinctly represents the characteristics of the data. Raw data are, in many cases, in a poorly structured form [12]; therefore, data pre-processing is required prior to data analysis. Even after reformatting, the data may be incomplete in many ways: some features may be redundant or unnecessary and, most importantly, original features still demand considerable manipulation before prediction. Key learnings from the data are mixed or hidden in several features, and so generating new features based on multiple features to extract the true nature of the data is crucial.

However, feature engineering today is mainly a manual and tedious job requiring considerable trial and error [16]. Since each dataset is domain-specific, automating any transformation process is difficult. The automation is time-consuming even for data analysts who are proficient in programming, making it extremely difficult for domain experts who are non-programmers to perform these types of tasks. Domain experts often know how the data should look, but they do not have sufficient skills to write the code to achieve the transformation.

Figure 1 describes a typical data transformation scenario using the Titanic dataset, taken from Kaggle. This dataset describes passengers and their properties, and data scientists try to predict the passengers' survival rates based on their properties. In the dataset, a feature named "Cabin" indicates the room number of each passenger. Most of the data are comprised of a letter followed by a number (e.g., "C231"), some are comprised of several room numbers (e.g., "A21 A22"), and some data are missing (e.g., "None"). Directly feeding this raw data into a classifier leads to sub-optimal predictions, so pre-processing is necessary. One example of a data cleaning process is as follows. First, we replace all missing data with an alternative value. Then, we categorize the data based on the first letter (e.g.,

\footnotetext{
${ }^{1}$ https://www.kaggle.com/francksylla/titanic-machine-learning-from-disaster
} 
"C23" belongs to category "C"). For special cases, such as when data have two or more room numbers, we choose one letter to unify the data format (e.g., "A21 A22" to category "A"). Such procedures are generally too complex to be executed without programming skills when working on large datasets. If data analysts with limited or no coding skills cannot generate features by themselves, they can rely on existing data analytics tools such as Weka [8] However, such applications are generally insufficiently user-friendly for novice users, and their capability is limited.

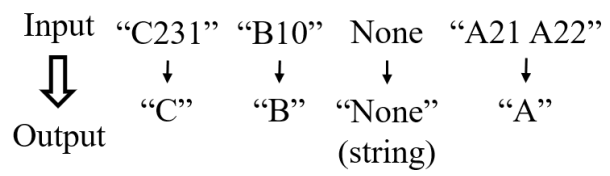

Figure 1: Example of data transformation in Titanic dataset

Programming by example (PBE) may be a powerful technique to enable non-programmers to easily process data. FlashFill [6], for example, learns string transformation rules called domain-specific language (DSL) from input-output examples provided by users. PBE enables users to reformat or transform unstructured data without requiring any programming knowledge, and some data transformation systems using PBE have been proposed for this purpose [3, 6, 9, 12, 24]. FlashFill [6] synthesizes an algorithm for domain-specific language, $L$, from a small number of examples using version-space algebra [18] to succinctly represent a large set of programs in L. Although these techniques are useful for nonprogrammers, their use is not sufficiently widespread because of the lack of usability and confidence in the correctness of the synthesized programs [17]. In fact, FlashFill does not have any functions to resolve ambiguity.

FlashProg [19] is a program-centric approach for disambiguation where the system shows synthesized programs to the user and lets them select a desirable one. The top five ranked programs are described in natural language, and users can select the one that best describes their intention. Users can also compare two programs by observing their outputs and picking a desirable one. One problem with such a program-centric approach is that it is still difficult and tedious for non-programmers to read the natural language explanations of the synthesized programs. One must understand the logic explained in natural language, which requires extra effort. Another problem is that PBE systems tend to synthesize variations that are too many and similar (see Section 5), which makes it tedious for the user to choose correctly.

We propose a data-centric interaction approach for disambiguation in PBE systems to address the problems of program-centric approaches. Users focus on processing a given dataset and examine and modify the candidate outputs rather than the synthesized programs. Using the user-provided examples, the system first generates candidate programs and then applies all the programs to the dataset to create a list of candidate outputs for each data cell. If the candidate outputs are incorrect, users can directly modify them and create their preferred features. We expect that examining program outputs will be easier and more intuitive than examining the programs. We implemented a prototype system, FlashAttention, to demonstrate the effectiveness of the data-centric disambiguation with three key features: cell coloring, a colored scroll bar, and checkboxes. Our approach allows users to process complicated data in an intuitive and direct way without being overwhelmed by the underlying programs; therefore, we expect it to be especially beneficial for non-programmers. To evaluate our approach, we conducted a user study with non-programmers using a combination of PROSE benchmarks [7], Kaggle datasets, and scraped data. This paper makes the following key contributions:

- We present a novel interaction design - data-centric disambiguation - for data transformation using PBE. It focuses on efficiently transforming a given dataset rather than on the completeness of the synthesized programs.

- We present a user interface called FlashAttention that uses cell highlighting, an augmented scroll bar, and checkboxes as an example of a data-centric interaction for data transformation.

- We evaluate our system and show that our interface successfully guides users to efficiently and confidently perform data transformations.

\section{RELATED WORKS}

Data Cleaning and Integration- To date, many algorithmic techniques for supporting data cleaning and integration have been proposed, such as erroneous value detection [10], information extraction [25], and schema matching [21]. Our system makes these techniques intuitively accessible to non-programmers.

Interactive Data Transformation- Several interactive systems assist users in data cleaning. Toped ++ [23] is an interface for creating topes: objects that support data validation and transformation. Potter's Wheel [22] is an interactive data cleaning system that provides a transformation language for data formatting and outlier detection. Ajax [5] facilitates data cleaning and transformation programs. Wrangler [13] provides an interactive system for data cleaning and transformation, automatically suggesting relevant transformations based on the context of user interactions. Although these systems help the users having limited programming backgrounds to transform data, users still need to properly choose transformation operators in the correct order, which seems too challenging for non-programmers.

Programming-by-Example (PBE)- Some studies tackle the usability issue using PBE techniques. SWYN [3] constructs regular expressions from text selection examples provided by users and shows visual representations to help users evaluate the effect of the expressions. FlashFill [6] and BlinkFill [24] construct directed acyclic graphs (DAG) using regular expressions to infer string transformation rules from input-output examples. Similar PBE-based systems that support data transformation also exist $[9,12]$. These systems do not require users to have prior programming skills. Our system makes these PBE-based techniques easily accessible for non-programmers using interactive visualization.

Automatic Feature Generation- Recently, several works aiming at automating feature generation have been proposed. First, Data Science Machine [14]was proposed to automatically generate features by searching for all possible new features using various 
operators. Similarly, ExploreKit [15], Cognito [16], and LFE [20] were proposed for automatic feature generation in data analysis. Although these systems reduce the burden of data analysis by automatically generating useful features for prediction, they require considerable computation time (e.g., the upper runtime limit of ExploreKit is three days) and none of them support string transformation. Our system supports string transformation and our data-centric framework itself can be extended to arbitrary types of feature generation.

Visualization Using Scroll Bars- Several augmented scroll bars have been proposed in the context of information retrieval. Attributemapped scroll bars [4] use colored marks in the scroll bar to highlight the salient properties in a document. Edit wear and read wear [11] graphically visualizes the edit history of the document and marks how often the sections of the document have been read. They used an augmented scroll bar for a similar purpose as our interface (i.e., to highlight important information), but our study is the first to propose using this function in the context of PBE.

FlashProg- Our system shares the goal with FlashProg in that both support disambiguation in PBE. The fundamental difference between the two is that they focus on synthesized programs while we focus on program outputs. We believe that these two approaches are complementary. One can use either of the two approaches or combine them in an application. There is also an important difference between the two systems. The target task of their system is data extraction (take a long document as input and highlight specific parts), while the target task of our system is data transformation (take short text strings as input and convert them to another text strings). So, it is not possible to run direct head-to-head comparison between the two systems. One needs to implement a data-centric data extraction system or a program-centric data transformation system, but we leave it as a future work.

\section{USER INTERFACE}

This section describes FlashAttention from the users' point of view. FlashAttention is a PBE-based interactive system for data transformation. Users can quickly process various types of data without writing a single line of code. Figure 2 shows a screenshot of FlashAttention. Using the toolbar (Figure 2a), users can import and save data. This worksheet (Figure $2 b$ ) is the main part of our system. The description window (Figure 2c) informs users about the current status of the transformation process. The PBE calculation runs when the user clicks the run button (Figure 2d) or presses the return key. Users can also undo a procedure by clicking the undo button.

Figure 3 describes a typical workflow using our system. We currently support the transformation of a single feature. The original data are placed in the first column ("Input"), and users can provide a user-defined example in the second column ("Output"). In this example, users want to extract the first letter $(\mathrm{A}-\mathrm{Z}, \mathrm{a}-\mathrm{z})$ from the room numbers. (i) The users first provide an input-output example (in this case, "C21" to "C") to show their preference. FlashAttention runs PBE and shows the users the most plausible outputs based on the examples. Now, the user can examine and interact with the data in the following three ways: (ii) Ambiguous cells are highlighted using color, and users can easily determine whether the system is confident in the current output. (iii) Users can also use

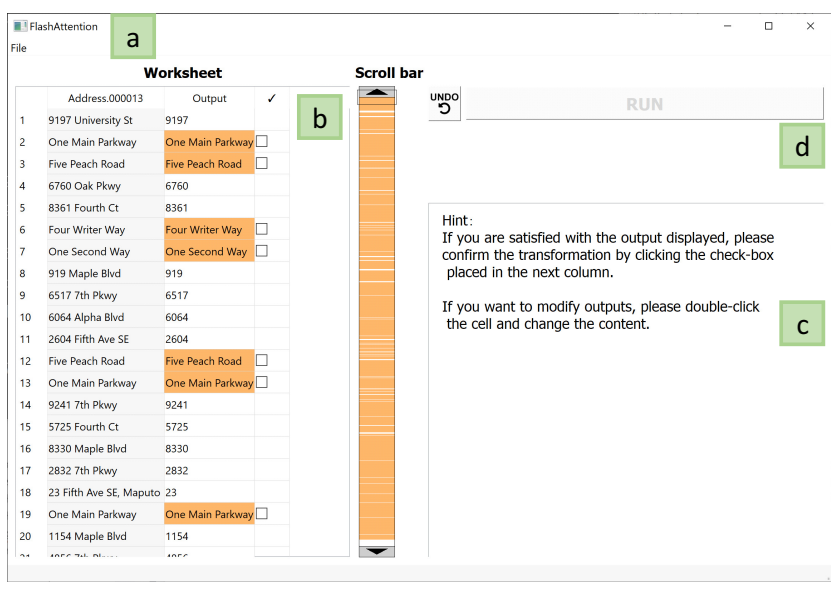

Figure 2: A screenshot of the prototype of our user interface. In this example, the user provided one input-output example (“9197 Universiy St" to “9197"), and clicked checkboxes twice (“6760 Oak Pkwy" to "6760" and "6517 7th Pkwy" to “6517”). a- Toolbar. b- Worksheet (interaction space), c- Description window, $d-$ Run and undo buttons.

the augmented scroll bar to easily see which part of the dataset is still ambiguous. (iv) If they are satisfied with the current output, they can click the corresponding checkbox to confirm the transformation. After examining the data, users can either (v) provide another input-output example (in this case, blank to "None") or (vi) confirm the current transformation by clicking the corresponding checkbox. Users repeat steps (ii)-(vi) until all the predictions are satisfactory.

\section{IMPLEMENTATION}

Data-centric interaction is a novel interaction design paradigm for disambiguation in PBE systems, where users examine and modify candidate outputs rather than the synthesized programs. In PBE systems, the number of programs is typically approximately $10^{30}$ [19]; further, even if programs are succinctly represented using versionspace algebra, the number of programs may still easily surpass 1000 . In such cases, program-centric approaches force users to compare numerous similar programs. Assume we have address data and want to extract the first part of the street number:

"2117 Hartway Street" $\rightarrow$ "2117."

Here, more than 30,000 programs are consistent with the user's specification. The top-ranked programs are very similar, and comparison can be very tedious, especially for non-programmers. Ambiguity occurs when we apply synthesized programs to other parts (or cells) of the data. Thus, by applying all candidate synthesized programs to each cell, we can obtain the list of candidate outputs for each cell; the number of outputs on this list is far less than the number of synthesized programs. By focusing on the candidate output list rather than programs, we can drastically reduce the number of candidates that should be compared to resolve the ambiguity in the data. In FlashAttention, we only use this ambiguity as an indicator of potential transformation errors. That is, a cell is 


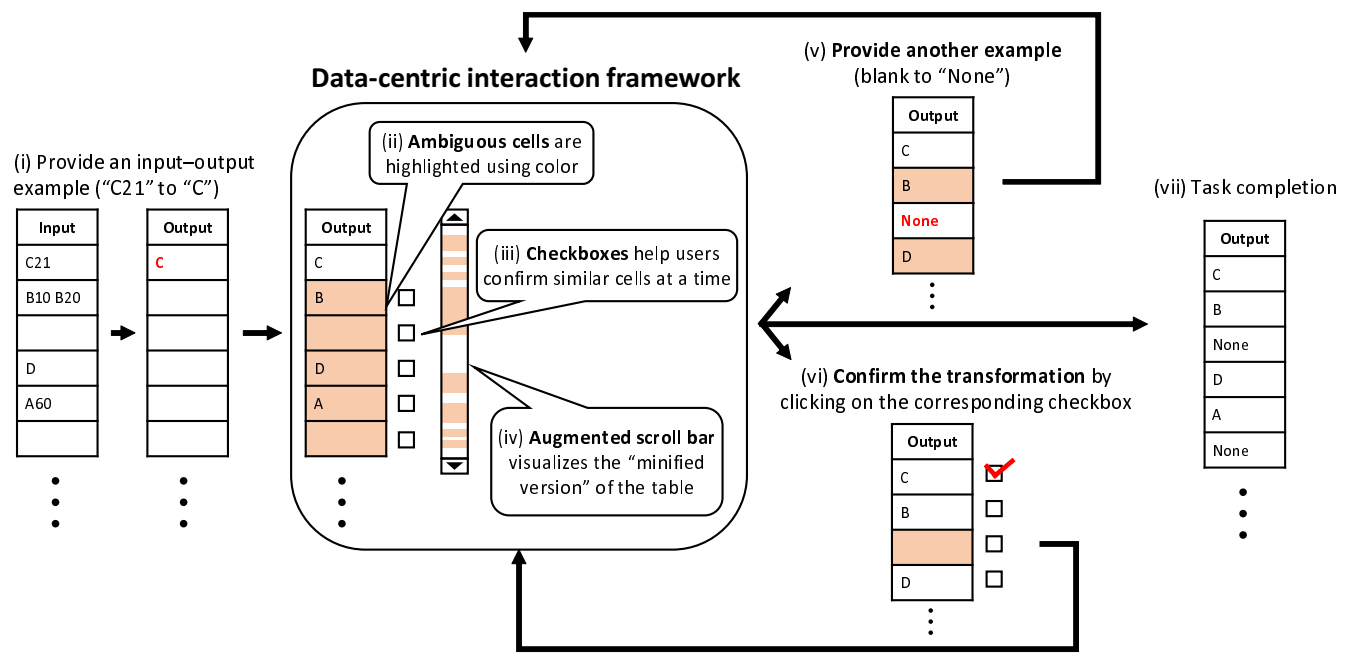

Figure 3: The FlashAttention workflow. (i) Users provide an input--output example (e.g., "C21" to “C”). Users can interact with the data in three ways in the data-centric interaction framework: (ii) cell coloring, (iii) augmented scroll bar, and (iv) checkboxes. After examining the data, users can either (v) provide another example or (vi) confirm the transformation by clicking on the corresponding checkbox. Users repeat this process until (vii) all data align with the users' preferences.

highlighted when it has ambiguity, and users modify the output if they are unsatisfied. This drastically reduces the burden on users, as they do not have to compare different outputs.

Leveraging the advantages of the data-centric disambiguation approach, we propose FlashAttention, a user interface that helps users resolve ambiguity and correct incorrect outputs. In this study, we propose the following three core graphical user interface components: cell coloring, an augmented scroll bar, and checkboxes.

\subsection{Synthesis Algorithm}

The synthesis algorithm used in our system is the same as in FlashFill [6]. The synthesis algorithm comprises the following functions: generate, intersect, and ranking.

Generate: enumerates all the possible trace expressions that map each input to the output in user-defined examples.

Intersect: intersects the enumerated trace expressions and constructs top-level conditionals to synthesize a program that can handle all the input-output examples.

Ranking: chooses the synthesized program that best describes how the user wants the output to look based on a ranking criterion.

Trace expression is a concatenation $\left(\mathrm{f}_{1}, \ldots, \mathrm{f}_{\mathrm{n}}\right)$ of atomic expressions $\mathrm{f}_{\mathrm{x}}$, where $\mathrm{f}_{\mathrm{x}}$ is a constant-string ConstStr, a substring SubStr, or a loop expression Loop. Each of these functions is defined in [6]. Note that our system eliminates unpromising programs to reduce calculation time. We follow FlashFill's approach, assuming that the smaller and simpler explanation is usually the correct one. We prefer that the conditionals and trace expressions have fewer arguments. Then, we rank the candidate programs using a scoring function. We prefer tokens (regular expressions that match a part of a string) corresponding to a larger character class to prioritize generality (as in FlashFill).

\subsection{Data-centric disambiguation}

After obtaining a set of valid synthesized programs, we apply them all to every input and create a list of candidate outputs. Then, we sort the outputs in each row from left to right based on the ranking criterion. Suppose we have a single feature comprising six rows: $\{\mathrm{C} 21, \mathrm{~B} 10 \mathrm{~B} 20$, None, $\mathrm{D}, \mathrm{A} 60$, None $\}$. On providing an input-output example $\mathrm{C} 21 \rightarrow \mathrm{C}$, we get the candidate outputs, as shown in Figure 4. Then, we select the cells to be colored. The details of the coloring function are described in the next subsection. The visualization is interactive, and each time a user provides more information to the system, it synthesizes the programs again or only retains the programs that are in accordance with the user's preference. More specifically, when a user modifies a cell and the output example is included in the list of candidate outputs, the system just discards the synthesized programs that are inconsistent with this new input-output example. This process reduces the computational time and thus makes the entire approach more efficient. If the user's input is not included in the candidate outputs, the system resynthesizes programs.

\begin{tabular}{|l|l|l|l|}
\hline \multicolumn{1}{|c|}{ Input } & Output 1 & Output 2 & Output 3 \\
\hline C21 & C & & \\
\hline B10 B20 & B & BB & C \\
\hline None & & & \\
\hline D & D & C & \\
\hline A60 & A & C & \\
\hline None & & & \\
\hline
\end{tabular}

Figure 4: Example of multiple candidate outputs.

One of the key insights behind our approach is that "we do not always necessarily narrow down to a single program." In most practical cases, as long as all cells are accurately processed according 
to the user's preference, it is acceptable if the adopted program is slightly different from the user's initial expectations. For example, when we extract numbers from phone number data, we are unconcerned about whether the synthesized program is "Extract all numbers if there are at least two hyphens (-)" or "Extract all numbers if there is at least one hyphen (-)" because both programs successfully extract all numbers. Of course, in some situations, obtaining a precise rule based on user specifications is critical, such as building the final version of a prediction model in a machine learning task; however, in many cases, users only want to process the data at hand, such as in building prototype models in data analysis and formatting internal company data. Our approach prioritizes the efficiency and simplicity over the completeness or generality of the synthesized program, and we argue that this approach is especially suited to non-programmers.

4.2.1 Coloring Cells. We highlight ambiguous cells to help users identify which need additional processing. To resolve ambiguity, FlashProg [19] compared two approaches: directly comparing different synthesized programs and answering example-based queries. Users showed a slightly higher preference for the latter because they were more confident in the result. Our cell-coloring also works as an example-based query, but it is more efficient than asking questions one by one because users can compare different outputs at a glance as they are all displayed in one table.

Cells are defined as ambiguous if they have multiple candidate outputs or if no potential outputs were found based on the current user's input-output examples. Users can immediately understand which inputs are ambiguous because all such cells are highlighted. The highlighting guides users toward the next action and helps them efficiently resolve ambiguity. Ambiguous cells can be resolved by either (1) confirming them using a checkbox, as described later, or (2) running PBE again by directly entering the correct output if the users are not satisfied with the current output.

4.2.2 Augmented Scroll Bar. The augmented scroll bar next to the table helps users in not only going back and forth between cells but also seeing which parts require further processing by highlighting ambiguous cells. Users can jump to a given cell by clicking their corresponding position in the scroll bar. This enables users to identify ambiguous cells at a glance and quickly fix them, even if there is only one cell. This should help to prevent users from overlooking incorrect outputs. Our augmented scroll bar is similar to the bird's eye view in FlashProg, but theirs only displays the preview of the result without considering ambiguity. In contrast, our augmented scroll bar highlights ambiguous outputs to help the user quickly find problematic outputs.

4.2.3 Checkboxes. Check-boxes help users confirm all the ambiguous cells with similar inputs with a single click. In many cases, although many ambiguous cells exist, their variance is essentially small. For example, a feature called Cabin in the Titanic dataset has 687 blank cells, which comprise $77 \%$ of the features. Users can confirm $77 \%$ of data transformation by confirming a blank cell once. The checkbox function supports confirmation of similar cells.

Checkboxes are only set for ambiguous cells and are not placed next to unambiguous cells, where ambiguity is already resolved. Upon the user's confirmation, the system completes the other cells with inputs similar to the confirmed cell. This process is performed by discarding the synthesized programs that are processing the inputs in different ways. For example, when a user confirms a process "transform blank cells to string 'None'," the system only leaves the synthesized programs that transform blank cells to "None" and removes all other programs. Users repeat this confirmation process until only the programs aligning with the users' preferences remain. Multiple programs may remain in the end, but we do not have to curtail the list to a single program because they all satisfy the users' preferences. This is one of the key points of the datacentric disambiguation approach.

\section{PRELIMINARY EXPERIMENT}

We ran a preliminary experiment to evaluate the effectiveness of our data-centric approach. We compared the number of synthesized programs and candidate outputs. We anticipated that the number of candidate outputs would be much fewer than the programs and thus easy for users to examine and verify. We used six features in total. Two features came from Kaggle [2], one was scraped from a website [1], and the last three were from FlashFillTest [7]. For each feature, one data point was selected as input, and a correct output was fed to the system. The system then synthesized programs and applied them to all the data points. We then counted the number of candidate outputs for each data item and took the maximum of the counts. Note that we only fed one input-output example for each feature, and thus the data transformation itself was incomplete. Table 1 shows the results; the number of programs grows rapidly when the input-output example is complicated, while the maximum number of candidate outputs remains relatively small. Although our system only displays the most plausible candidate output, the results imply that users only need to consider a limited number of possible choices if they focus on the outputs.

Furthermore, it appears that the top-ranked programs often look quite similar when the feature is complicated. For example, for "DateTime:000005", 2,806 programs were generated. The top-ranked DSL was "if there is a number, the first number is extracted," while the second-highest ranked was "if there is a hyphen (-), the first number is extracted." The third-highest ranked was "if there are two numbers, the first number is extracted." All were similar and made no difference to the outputs. In contrast, our system only highlighted the data points that were critical in completing the given data transformation. The user could directly modify or confirm the output, which was probably more intuitive and could be more efficient for non-programmers than examining the programs. The results suggest that our data-centric approach is an effective way to interact with users to resolve ambiguity.

\section{USER STUDY}

We conducted a user study to compare the performance of our FlashAttention with that of FlashFill. We hypothesized that FlashAttention is more efficient (faster and leading to fewer errors) than FlashFill when working on large datasets. We also hypothesized that such differences are more evident for larger datasets. We evaluated these two systems based on their completion time for each data transformation task as well as the number of errors and a post-task questionnaire. 


\begin{tabular}{|c|c|c|c|}
\hline Feature name & Input-Output Example & Programs & Candidates \\
\hline Cabin [2] & C123 $\rightarrow$ C & 34 & $\mathbf{3}$ \\
\hline Ticket [2] & A/5. 2151 $\rightarrow$ A & 86 & $\mathbf{5}$ \\
\hline Match up [1] & TOR @ GSW $\rightarrow$ @ & 8 & $\mathbf{2}$ \\
\hline Abbreviation.000001 [7] & Nihil Impedit $\rightarrow$ NI & 13 & $\mathbf{3}$ \\
\hline Column.000001 [7] & Coln8 $\rightarrow$ Col8 & 114 & $\mathbf{1 0}$ \\
\hline DateTime.000005 [7] & 2026-12-30T $\rightarrow$ 2026 & 2806 & $\mathbf{1 6}$ \\
\hline
\end{tabular}

Table 1: The number of synthesized programs and the maximum number of candidate outputs for each feature. In the second column, the left-hand side denotes a raw input, and the right-hand side is a corresponding output expected for this input. "Programs" denotes the number of programs generated by feeding the example. "Candidates" means the maximum number of candidate outputs for the inputs in the feature generated by this example.

\subsection{Experimental Design}

We used a $2 \times 3$ mixed design with two between-subjects conditions and three within-subjects conditions: METHOD (FlashAttention or FlashFill) and DATASET SIZE (small, medium, or large). Using one of the two interfaces, participants performed four tasks in each of the three DATASET SIZE conditions-that is, a total of twelve tasks.

\subsection{Procedure}

Participants first attended a training session for the system they evaluated. A scripted demonstration showed how to provide output examples and confirm the transformation by clicking a checkbox. After the demonstration, the participants were asked to work on the same feature as demonstrated. They could also work on up to two additional practice tasks until they completely understood how the system worked. These features were not used in the main task.

Next, the participants worked on the main task set. The task set was the same for all participants. The main task set comprised 12 tasks, and each task dealt with the transformation of a single column. They began with the first task and moved on to the next task upon completion. Although they were allowed to skip tasks, they were not allowed to return once they had proceeded to the next task. The skipped task was recorded and marked as an error. The participants were given a short break between tasks to remain focused on each task. The maximum time for each task was limited to 90 seconds (excluding the time to read the task description). If a user reached this limit, the transformed outputs were considered the final output. After completing all the tasks, each participant was asked to answer a post-task questionnaire designed to evaluate the system they used in terms of efficiency and confidence in the results. A 7-point Likert scale (1-7) was used, with 1 being the least useful/confident. The questions are shown in Figure 7. The participants were also asked about the most unpleasant and pleasant aspects of the interface. For participants who used FlashAttention, additional questions were asked using the Likert scale that specifically addressed each feature in the system. The questions are shown in Figure 8. The experiment took approximately 60 minutes per participant.

\subsection{Apparatus}

To evaluate the proposed method, we created a custom dataset using publicly available data. Of 12 datasets comprising the main dataset, 7 came from Kaggle ${ }^{1}$, 3 were taken from a public benchmark called FlashFillTest [7], and the remaining 2 were scraped from websites for ecological validity ${ }^{2}{ }^{3}$. FlashFillTest has 354 realworld/synthesized benchmarks for string-to-string transformation. Since we focus on string transformations in this study, we selected syntactic transformations (such as extracting a sub-string) from the benchmark and then selected three datasets.

Moreover, we pre-processed the full dataset and included additional items, making the difficulty of each task as equitable as possible. The appendix provides the details of this pre-processing. In doing so, the small dataset (the first four subset datasets) had approximately 300 items per column, the medium dataset (the next four) had approximately 3,000 items, and the large dataset (the last four) had approximately 30,000 items. This allowed us to evaluate the usability of the interfaces for different dataset sizes. Moreover, each dataset size had two difficulty levels: easy and difficult. The easy dataset could be processed without error by providing a few representative examples. The difficult dataset included a small number of exception cells that were artificially introduced to trigger mistakes (details of how we prepared these cells are presented in the appendix).

\subsection{Participants}

We recruited 26 participants for the study. Since FlashAttention is designed to support non-programmers in processing complicated data transformations, we recruited non-programmers who had some prior data processing experience. Of the 26 participants, 12 processed data every day, and 14 participants processed data several times a week. All participants had experience with manually processing data using general data processing tools, such as Microsoft Excel. Fifteen participants had used pre-defined functions in Microsoft Excel (e.g., "= Q1 + R1") and four had previously written macros in Microsoft Excel. None of the participants had processed data using programming languages such as Python or Ruby. We recruited participants using a company that provided business process outsourcing services. We divided the participants into

\footnotetext{
${ }^{1}$ https://www.kaggle.com/francksylla/titanic-machine-learning-from-disaster, https://www.kaggle.com/devvret/brazil-real-estate-listings, https://www.kaggle.com/jackywang529/toronto-bikeshare-data, https://www.kaggle.com/himanshupoddar/zomato-bangalore-restaurants, https://www.kaggle.com/russellyates88/suicide-rates-overview-1985-to-2016 ${ }^{2}$ https://stats.nba.com/players/boxscores/

${ }^{3}$ https://gemini.com/auction-data
} 
two groups, ensuring that the data processing experiences in each group were balanced. Participants in Group A used FlashAttention, whereas participants in Group B used FlashFill.

\subsection{Results}

We evaluated our approach based on task completion time, error ratio, and the post-task questionnaire. For the task completion time and error ratio, we present the results from a two-way mixed-design analysis of variance (ANOVA) with METHOD as between-subjects factors and DATASET SIZE as within-subjects factors. We conducted a Mann-Whitney U-test for the 7-point Likert scale questionnaire.

6.5.1 Task completion time. Figure 5 shows the average time required to complete one task, which is the average of four tasks in each dataset. Dark red and blue bars represent the computation time (program synthesis and visualization), and the light colors represent the required time, excluding the computation time. The combined dark and light colors represent the total time. Overall, our interface reduced the time required to complete tasks on average, $45.01 \mathrm{~s} \mathrm{(SD}$ $=14.78)$ compared with $65.96 \mathrm{~s}(\mathrm{SD}=24.46)$ (without calculation time). This difference was significant, as seen in the main effect of METHOD on the required time $\left(F_{1,48}=25.64, p=5.59 \times 10^{-20}\right)$. The difference was also significant if we included computation time $\left(F_{1,48}=13.25, p=9.83 \times 10^{-12}\right)$. No significant main effects of DATASET SIZE and their intersection were found.

We then ran a Tukey's honestly significant difference (HSD) test for the post-hoc analysis; Figure 5 shows the result indicated by * (representing statistically significant differences, $p<0.05$ ). The required time for FlashAttention was significantly shorter than that required for FlashFill in the medium and large datasets $(p<$ 0.05 ), and a significant difference in required time was seen in the small dataset $(p>0.05)$ (without computation time). When the computation time was included, the difference was significant for the medium $(p<0.05)$, and not significant for the small and the large datasets $(p>0.05)$. For the large dataset ( 30000 items), our interface required $38 \%$ less time, and for the medium dataset ( 3000 items), it required $45 \%$ less time than FlashFill. This indicates that FlashAttention successfully decreases the time required to perform data transformation.

The results show that the small dataset required the longest time of the three. A possible reason for this is that the small data set was processed first in the user study, and the participants were not used to operating the system. Another possible reason is that for the large dataset, it took longer to display the result, and so the users had more time to think about the next step to take while waiting for the result to be displayed.

6.5.2 Task error ratio. We counted the number of errors in each trial. We used the same technique as used in FlashProg [19]. We selected the same 32 representative error types that could occur during the transformation task and counted the number of error types that appeared in the results. This process is more reasonable than simply counting the number of incorrect outputs. An example of a representative error is forgetting to fix missing values.

Figure 6 shows the error ratio of each data set size (small, medium, and large). As a result, our interface reduced the number of errors

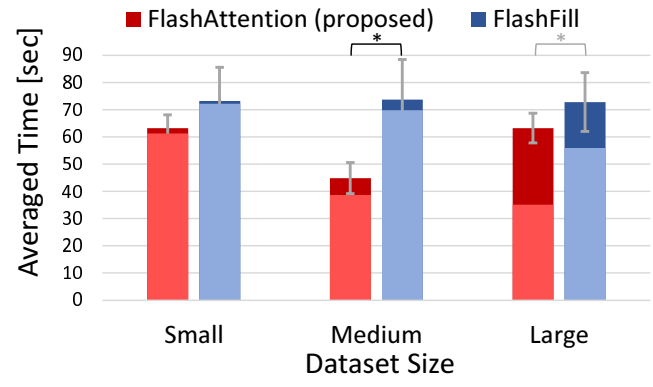

Figure 5: Task completion time. The small dataset has approximately 300 rows, the medium dataset has approximately 3000 rows, and the large dataset has approximately 30000 rows per task. The error bar displays a $95 \%$ confidence interval. Each category contains four tasks, and the time displayed above is the average time required to complete the four tasks. Dark red and blue represent computation time, and light red and blue represent the time excluding the computation time.

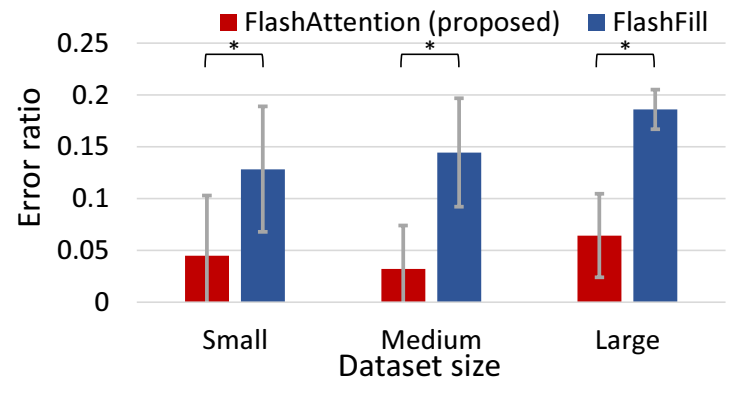

Figure 6: Task error ratio. Small $=\mathbf{3 0 0}$ rows, medium $=\mathbf{3 0 0 0}$ rows, and large $=30000$ rows. Each category contains four tasks, and the result above is the average error ratio of the four tasks. The error bar displays a 95\% confidence interval.

on average, $4.7 \%(\mathrm{SD}=0.088)$ compared with $15.3 \%(\mathrm{SD}=0.091)$. This difference was significant, as seen in the main effect of METHOD on the error ratio $\left(F_{1,48}=29.99, p=5.16 \times 10^{-22}\right)$. No significant main effects of DATASET SIZE and intersection were found. We then ran the Tukey's HSD test for a post-hoc analysis, and Figure 6 shows the results indicated by * $(p<0.05)$. The error ratio was significantly lower than that in FlashFill for all dataset sizes. Using FlashFill, participants made two times more errors than when they used FlashAttention (12.8\% compared with $4.49 \%$ in the small, $14.4 \%$ compared with $3.21 \%$ in the medium, and $18.6 \%$ compared with $6.41 \%$ in the large datasets). This indicates that FlashAttention helps prevent users from making mistakes while working on data transformation tasks.

6.5.3 Post-task questionnaire. The results of the post-task questionnaire are shown in Figure 7. For each question, the participants were asked to answer on a 7-point Likert scale. Overall, the figure indicates that the participants preferred using our interface for most aspects. 


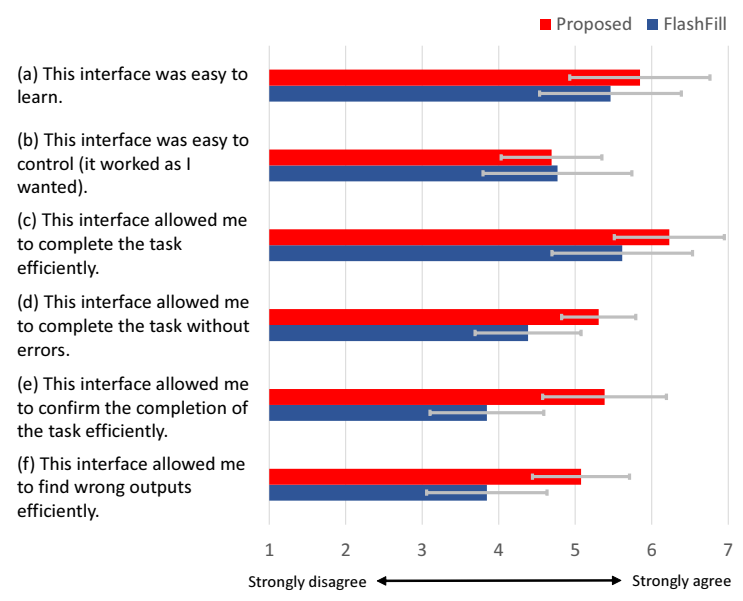

Figure 7: Post-task questionnaire. The error bar displays a 95\% confidence interval.

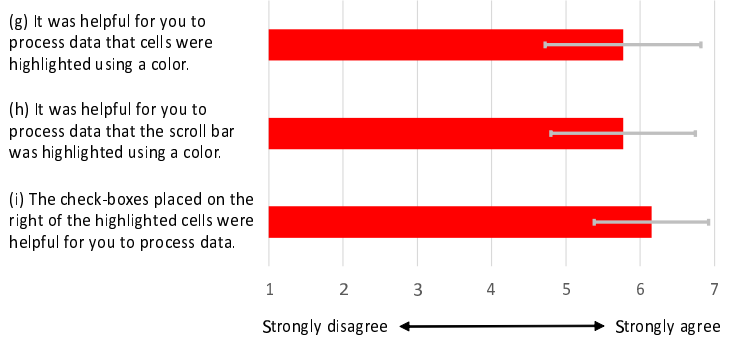

Figure 8: Post-task questionnaire 2. The error bar displays a 95\% confidence interval.

For the first and second questions, the mean scores were high and almost the same (in (a), our interface scored 5.85 and FlashFill scored 4.69; in (b), our interface scored 5.46 and FlashFill scored 4.77). The difference was not significant ( $p=0.54, r$ (effect size $)=0.17$ ). This indicates that both were easy to control and understand.

The participants found FlashAttention more efficient in completing tasks without errors, which is apparent from questions (c), (d), and (e). In both interfaces, participants considered the efficiency of the data transformation as an advantage of the interface. Participants who used FlashAttention also reported additional advantages. For example, they said the accuracy of the transformation was higher than if they had manually performed it independently. They also mentioned that the highlighting was helpful in checking the transformation result and that the interface helped them find incorrect outputs.

Overall, the participants felt more confident in their results when they used FlashAttention. For question (c), FlashAttention scored 6.23 and FlashFill scored 5.62 on average. For question (d), FlashAttention scored 4.38 and FlashFill scored 5.31 on average. However, these results are not significant (for (c), $p=0.26, r=0.31>0.30$; for (d), $p=0.18, r=0.37>0.30)$. Most of the participants indicated that the colored cells and the augmented scroll bar helped them find incorrect outputs; these two functionalities may have contributed to increasing the participants' confidence.

Our interface helped the participants feel confident that they had completed the task (for question (e), our interface scored 5.38 and FlashFill scored 3.85 on average). The difference was significant $(p<0.05, r=0.55>0.50)$. Participants also felt that our interface was more helpful in efficiently finding incorrect outputs (for question (f), FlashAttention scored 5.08 and FlashFill scored 3.85 on average). However, the result was not significant $(p=0.08$, $r=0.49>0.30$ ). One of the major drawbacks of FlashFill was the lack of information about the current transformed outputs. From the participants' feedback, FlashFill "takes time to check whether all of the cells have the correct outputs," and "if there is a way to check the outputs efficiently, that would be better." FlashAttention successfully reduced this burden by presenting rich visual information about ambiguity.

Participants in Group A (FlashAttention) were also asked additional questions regarding each FlashAttention function (coloring cells, the augmented scroll bar, and checkboxes). Figure 8 shows the results.Most of the participants agreed that all functions were helpful (>4), and no respondents rated them less than 4 on the 7-point Likert scale.

They were also asked to comment on each function of the proposed system. In general, they answered that cell coloring was helpful (the mean score was 6.15). Examples of positive comments included: "It was easy to distinguish unchecked cells from the ones that have already been processed," and "I was able to intuitively understand that colored cells contain different types of information." No negative comments were made regarding cell coloring. Moreover, the suggestions from some participants who used FlashFill also supported the usefulness of cell coloring. They said that "having a function that colors blank cells" or "an efficient way to check the outputs" would make FlashFill more useful, which is exactly what our coloring function supports.

The augmented scroll bar was useful for efficient interactions (the mean was 5.77). This augmented scroll bar offered two main advantages: the ability to immediately jump to ambiguous cells and the ability to quickly review the entire dataset. Out of 13 participants, 6 mentioned the former point and 7 reported the latter. Positive comments included: "It was very efficient because I could quickly check which cells are processed yet and could immediately move to the corresponding cell by clicking on a colored region in the scroll bar," and "It was helpful to detect which cells were processed based on my last input, even if they are located around the bottom of the table."

The checkbox function was also useful. The mean score for question (i) was 5.77, which shows that the participants highly valued this function. The participants mainly recognized the following two points as advantages of the checkbox function: efficient data processing and the prevention of overlooking. Out of 13 participants, 9 mentioned that this function reduced the burden of checking the outputs because they could simultaneously confirm the transformation of many similar cells by clicking one checkbox. Additionally, two respondents said that the checkbox function helped them avoid overlooking incorrect outputs. The following are examples of comments regarding the checkbox function: "It was efficient because I 
do not have to check the cells that were correctly processed multiple times" and "The checkbox function was efficient because it processes similar cells by only clicking on a checkbox once and reduces the workload."

\section{DISCUSSION}

Overall, the user study showed the validity of our data-centric approach. The task completion time was significantly shorter in the medium and large datasets, and the error ratio was also significantly smaller than FlashFill's error ratio. The results of the questionnaire also validated the advantages of each function in FlashAttention. Therefore, FlashAttention is clearly more effective in efficiently performing error-free data transformations. The following further elaborates on specific topics.

Did the Data-centric Disambiguation Help Users to More Efficiently Resolve Ambiguity than FlashFill?

The post-task questionnaire ((d), (e), and (f)) and the lower error ratio in FlashAttention clearly show the validity of data-centric disambiguation. The colored cells and checkboxes highlight the ambiguity in the data; when no colored cells or checkboxes are visible, it is apparent that no ambiguity exists in the data. These strong signs have helped users spot potentially erroneous outputs and feel confident after resolving them.

On the other hand, it should be noted that if the user's intended program is beyond the expressiveness of the system's underlying DSL, the system might not output correct results. Users might overlook such errors because there is no warning from the interface. In this user study, the DSL was designed to express all the transformation rules required in the tasks, but issues may arise in real-world tasks. A participant said that occasionally undesired outputs were incorrectly confirmed. However, we suspect that he probably attempted an incorrect transformation since all tasks could be solved using the prepared DSL. A potential solution is to make the DSL sufficiently expressive, but this comes with the trade-off of increased program synthesis time. Another option is to interpret which transformation the users are attempting (e.g., syntactic transformation) and ask them if the proposed action aligns with their intention. These are the important observations revealed by the user study, and we expect future studies will address these problems.

\section{Will Non-programmers be able to Easily Operate this Sys- tem?}

The post-task questionnaire ((a), (b), and (c)) suggests that FlashAttention is easy to operate for non-programmers; it was easy to learn and control. Also, the error ratio was significantly lower than FlashFill's for all tasks. Moreover, six participants said that FlashAttention was easy to understand or convenient. Some participants said that they enjoyed the tasks: "I was able to operate as though it were a game" and "It was fun and convenient." These comments demonstrate that people without data transformation experience can also easily complete these tasks using FlashAttention.

On the other hand, some participants required more time to acclimate to the system than we expected, despite the three practice tasks, which were added as a result of the pilot study: "I was struggling at first, but as the tasks proceeded, I understood the system more and was able to proceed with the tasks more and more quickly" and "the system is easy to use once I get used to it." The increased time needed was probably attributable to their low familiarity with data analysis. Nevertheless, since most participants completed the tasks with fewer errors than those using FlashFill, and the participants expressed satisfaction in their comments and feedback, we can conclude that non-programmers can more easily and efficiently complete data transformation tasks using FlashAttention compared with FlashFill.

\section{Limitations}

Reliability of the outputs-Although our study mainly focused on visualization and the interactive interface and not on the PBE algorithms, note that the level of trust that users can place in the visualization result depends on the PBE results. A participant noted that "the ambiguity should not be resolved all at once if the patterns are entirely different," which implies that patterns perceived by humans might not always be in accordance with the system. Dealing with typographical errors-Currently, FlashAttention does not support detection of typographical errors; hence, when a user makes a typographical error, this erroneous transformation is automatically confirmed because it is treated as an input-output example and this is not highlighted. Combining our system with anomaly detection techniques to identify anomaly input-output examples would be an interesting direction to extend our approach. Time for displaying the outputs-For the large dataset, FlashAttention took 28 seconds on average for calculation, which is longer than FlashFill's calculation time. The underlying PBE algorithm is exactly the same as FlashFill's, and the complexity of the user's intended program did not change (i.e., a larger dataset does not mean the transformation rule is more complicated); the time difference is purely due to displaying the outputs in the interface. If the data size is huge (e.g., $>100,000$ rows), it might be possible that our system requires substantially more time to process, but the computational order of displaying outputs is still $O(n)$, and the algorithmic efficiency can be improved. Hence, this time difference does not disqualify the validity of our approach.

\section{Future works}

Our current implementation only supports single data transformations. Since generating new features using multiple features is a fundamental part of data analysis, developing support for multiple features is a key direction for future works. Besides, our current implementation focuses on string data extraction; it will be even more useful if it can deal with numerical data processing or syntactic transformation. Aside from the user interface, the system also has opportunities for improvement in terms of the underlying algorithm. For example, defining more efficient equations to calculate the ambiguity score is critical to ensure the reliability of the system. We also hope to integrate our system into various data processing applications and demonstrate its usefulness in practice.

\section{CONCLUSION}

In this study, we proposed a data-centric approach for data transformation, where users resolve the ambiguity in data by focusing on the predicted output rather than the underlying synthesized 
programs. Based on this paradigm, we created a novel interactive system called FlashAttention and evaluated our system with a user study. The results showed that highlighting ambiguous cells, showing them on an augmented scroll bar, and confirming similar cells by clicking checkboxes helped users to efficiently process data and increased their confidence in their transformation results.

\section{ACKNOWLEDGMENTS}

This work was supported by JST CREST JPMJCR17A1.

\section{REFERENCES}

[1] no date. NBA Advanced Stats: Players Box Scores. https://stats.nba.com/players/ boxscores/ Accessed: 2020-07-12.

[2] no date. Titanic: Machine Learning from Disaster. https://www.kaggle.com/ francksylla/titanic-machine-learning-from-disaster Accessed: 2020-07-12.

[3] Alan F. Blackwell. 2001. Your Wish is My Command. Morgan Kaufmann Publishers Inc., San Francisco, CA, USA, Chapter SWYN: A Visual Representation for Regular Expressions, 245-270. http://dl.acm.org/citation.cfm?id=369505.369521

[4] A. Wroblewski David, C. Hill William, and P. McCandless Timothy. 1990 Attribute-Mapped Scroll Bars. US.

[5] Helena Galhardas, Daniela Florescu, Dennis Shasha, and Eric Simon. 2000. AJAX An Extensible Data Cleaning Tool. In Proceedings of the 2000 ACM SIGMOD International Conference on Management of Data (Dallas, Texas, USA) (SIGMOD '00). ACM, New York, NY, USA, 590-. https://doi.org/10.1145/342009.336568

[6] Sumit Gulwani, William R. Harris, and Rishabh Singh. 2012. Spreadsheet Data Manipulation Using Examples. Commun. ACM 55, 8 (Aug. 2012), 97-105. https: //doi.org/10.1145/2240236.2240260

[7] Sumit Gulwani and Emilio Parisotto. 2018. PROSE Public Benchmark Suite https://github.com/microsoft/prose-benchmarks.

[8] Mark Hall, Eibe Frank, Geoffrey Holmes, Bernhard Pfahringer, Peter Reutemann, and Ian H. Witten. 2009. The WEKA Data Mining Software: An Update. SIGKDD Explor. Newsl. 11, 1 (Nov. 2009), 10-18. https://doi.org/10.1145/1656274.1656278

[9] Yeye He, Kris Ganjam, Kukjin Lee, Yue Wang, Vivek Narasayya, Surajit Chaud huri, Xu Chu, and Yudian Zheng. 2018. Transform-Data-by-Example (TDE): Extensible Data Transformation in Excel. In Proceedings of the 2018 International Conference on Management of Data (Houston, TX, USA) (SIGMOD '18). ACM, New York, NY, USA, 1785-1788. https://doi.org/10.1145/3183713.3193539

[10] Joseph M. Hellerstein. 2008. Quantitative Data Cleaning for Large Databases.

[11] William C. Hill, James D. Hollan, Dave Wroblewski, and Tim McCandless. 1992. Edit Wear and Read Wear. In Proceedings of the SIGCHI Conference on Human Factors in Computing Systems (Monterey, California, USA) (CHI '92). Association for Computing Machinery, New York, NY, USA, 3-9. https://doi.org/10.1145/ 142750.142751

[12] Zhongjun Jin, Michael R. Anderson, Michael Cafarella, and H. V. Jagadish. 2017. Foofah: A Programming-By-Example System for Synthesizing Data Transformation Programs. In Proceedings of the 2017 ACM International Conference on Management of Data (Chicago, Illinois, USA) (SIGMOD '17). ACM, New York, NY, USA, 1607-1610. https://doi.org/10.1145/3035918.3058732

[13] Sean Kandel, Andreas Paepcke, Joseph Hellerstein, and Jeffrey Heer. 2011. Wran gler: Interactive Visual Specification of Data Transformation Scripts. In Proceedings of the SIGCHI Conference on Human Factors in Computing Systems (Vancouver, BC, Canada) (CHI '11). ACM, New York, NY, USA, 3363-3372. https://doi.org/10.1145/1978942.1979444

[14] Max James Kanter and Kalyan Veeramachaneni. 2015. Deep feature synthesis: Towards automating data science endeavors. In IEEE International Conference on Data Science and Advanced Analytics (DSAA) (DSAA '15). IEEE, Paris, France. https://doi.org/10.1109/DSAA.2015.7344858

[15] Gilad Katz, Eui Chul Richard Shin, and Dawn Song. 2016. ExploreKit: Automatic Feature Generation and Selection. In IEEE 16th International Conference on Dato Mining (ICDM) (ICDM '16). IEEE, Barcelona, Spain, 979-984. https://doi.org/10. 1109/ICDM.2016.0123

[16] Udayan Khurana, Deepak Turaga, Horst Samulowitz, and Srinivasan Parthasrathy. 2016. Cognito: Automated Feature Engineering for Supervised Learning. In IEEE 16th International Conference on Data Mining Workshops (ICDMW) (ICDMW '16). IEEE Computer Society, Barcelona, Spain, 1304-1307. https://doi.org/10.1109/ ICDMW.2016.0190

[17] Tessa Lau. 2009. Why Programming-By-Demonstration Systems Fail: Lessons Learned for Usable AI. AI Magazine 30, 4 (Oct. 2009), 65. https://doi.org/10.1609/ aimag.v30i4.2262

[18] Tessa Lau, Steven A. Wolfman, Pedro Domingos, Pedro Domingos, and Daniel S Weld. 2003. Programming by Demonstration Using Version Space Algebra. Mach Learn. 53, 1-2 (Oct. 2003), 111-156. https://doi.org/10.1023/A:1025671410623
[19] Mikaël Mayer, Gustavo Soares, Maxim Grechkin, Vu Le, Mark Marron, Oleksandr Polozov, Rishabh Singh, Benjamin Zorn, and Sumit Gulwani. 2015. User Interaction Models for Disambiguation in Programming by Example. In Proceedings of the 28th Annual ACM Symposium on User Interface Software \&\#38; Technology (Charlotte, NC, USA) (UIST '15). ACM, New York, NY, USA, 291-301. https://doi.org/10.1145/2807442.2807459

[20] Fatemeh Nargesian, Horst Samulowitz, Udayan Khurana, Elias B. Khalil, and Deepak Turaga. 2017. Learning Feature Engineering for Classification. In Proceedings of the 26th International foint Conference on Artificial Intelligence (IFCAI'17). AAAI Press, Melbourne, Australia, 2529-2535. https://doi.org/10.24963/ijcai. 2017/352

[21] Erhard Rahm and Philip A. Bernstein. 2001. A Survey of Approaches to Automatic Schema Matching. The VLDB fournal 10, 4 (Dec. 2001), 334-350. https://doi.org/ 10.1007/s007780100057

[22] Vijayshankar Raman and Joseph M. Hellerstein. 2001. Potter's Wheel: An Interactive Data Cleaning System. In Proceedings of the 27th International Conference on Very Large Data Bases (VLDB '01). Morgan Kaufmann Publishers Inc., San Francisco, CA, USA, 381-390. http://dl.acm.org/citation.cfm?id=645927.672045

[23] Christopher Scaffidi, Brad Myers, and Mary Shaw. 2009. Intelligently Creating and Recommending Reusable Reformatting Rules. In Proceedings of the 14th International Conference on Intelligent User Interfaces (Sanibel Island, Florida, USA) (IUI '09). ACM, New York, NY, USA, 297-306. https://doi.org/10.1145/ 1502650.1502692

[24] Rishabh Singh. 2016. BlinkFill: Semi-supervised Programming By Example for Syntactic String Transformations. In PVLDB, 42nd International Conference on Very Large Data Bases (VLDB 2016). VLDB Endowment, New Delhi, India, 816827.

[25] Stephen Soderland. 1999. Learning Information Extraction Rules for SemiStructured and Free Text. Mach. Learn. 34, 1-3 (Feb. 1999), 233-272. https: //doi.org/10.1023/A:1007562322031

[26] D. K. Wind. 2014. Concepts in predictive machine learning. Master's thesis. Technical University of Denmark, Copenhagen, Denmark.

\section{A DATA}

In this study, we pre-processed the data for use in our user study.

\section{A.1 Augmentation}

Each FlashFillTest instance had approximately 5-10 input-output examples. Some of the scraped data also had a smaller dataset size than required. Since the dataset size was insufficient to test our system (we can manually process five cells in a few seconds), we augmented features to match the corresponding dataset sizes. Moreover, we discarded part of each example in some features due to time constraints. Some complicated features required a long time to process using PBE; therefore, we simplified them so that participants could finish processing in a limited amount of time. An example of a modification was to discard the latter part of address data ("2345 Redwood Road NE, Greensboro, VA 38891” to “2345 Redwood Road”).

The principles of the modification and augmentation were as follows:

(1) The required transformation process must be the same.

(2) The data distribution should be as similar as possible. We augmented the same number of data from each cell.

\section{A.2 Trimming}

Since we evaluated the dependency of the user's performance on the data set size, the size of each dataset had to be strictly controlled. A subset dataset was defined as a subset of the dataset where each column had the same data size as the others within the subset. To make subsets of the data, some larger datasets needed to be reduced in size. Data were sampled at fixed intervals until the specific data size was achieved that would avoid bias. 